\title{
Collaboration and competition on a wiki: The praxis of online social learning to improve academic writing and research in under-graduate students
}

\author{
Julie-Anne Carroll, Abbey Diaz, Judith Meiklejohn, Michelle Newcomb, and \\ Barbara Adkins \\ Queensland University of Technology, Australia
}

\begin{abstract}
While the Internet has been described as fundamental to higher education students, social and leisure internet tools are also increasingly being used by these students to generate and maintain their social and professional networks and interactions. Rapid technological advancements have enabled greater and faster access to information for learning and education. As such, we sought to integrate interactive, online social media into the assessment profile of a Public Health undergraduate cohort at the Queensland University of Technology (QUT). The aim of this exercise was to engage students to both develop and showcase their research on a range of complex, contemporary health issues within the online forum of Wikispaces (http://www.wikispaces.com/) for review and critique by their peers. We applied Bandura's social learning theory (SLT) to analyse the interactive processes from which students developed deeper and more sustained learning, and via which their overall academic writing standards were raised. This paper outlines the assessment task and the students' feedback on their learning outcomes in relation to the attentional, retentional, motor reproduction, and motivational processes outlined by Bandura in SLT. We conceptualise the findings in a theoretical model, and discuss the implications for this approach within the broader tertiary environment.
\end{abstract}

\section{Background and Rationale}

Web 2.0 has come to the forefront of higher education to improve learning amongst students in tertiary environments. The range of information and communication technologies that can be classified as web 2.0, such as blogs, social media, websites, and wikis, provide innovative and fertile learning spaces for students, as they shift the learning processes from linear pathways to more interactive and collaborative dynamics (Wheeler, Yeomans, \& Wheeler, 2008; Tétard, Patokorpi, \& Packalén, 2009). These tools also facilitate social learning, peer assessment, formative feedback from educators, and individual and group reflection on the learning experiences (Kirkwood, 2010; Knight, 2010). Studies have shown that the use of online tools, such as blogs and wikis, enhances students' engagement, deepens their learning experiences, and improves their overall academic achievement (Hemmi, Bayne, \& Land, 2009). Further to this, the inclusion of popular information and communication technologies in assessment items tends to heighten student interest in the topics being presented and engage them more readily in the tasks they are being set for learning purposes (Duffy \& Bruns, 2006).

While various online social media and technological mechanisms have been embraced within higher education environments, the evaluation and research pertaining to the use of wikis as educational tools is still in its infancy. However, it has been proposed that wikis do provide an opportunity for collaborative learning, for a deeper, more critical understanding of course content and enhancing motivation for academic achievement (Ducate, Anderson, \& Moreno, 2011; Feng \& Beaumont, 2010; Hutchinson \& Colwell, 2012; Yan, Tian, Vogel, \& Kwok, 2010). As such, the use of wikis within tertiary settings is slowing becoming prevalent across a variety of disciplines including teaching, foreign language studies and computer science, as they are thought to provide a space in which students can explore, reflect and provide critical analysis of their own and others' learning (De Wever, Van Keer, Schellens, \& Valcke, 2011; Ducate, et al., 2011; Hodgkinson-Williams, Slay, \& Siebörger, 2008; Ruth \& Houghton, 2009).

The most highlighted advantage of wikis as an educational tool is their capacity to engage students in collaborative learning. Wikis can facilitate a wide variety of authentic, collaborative and reflective learning activities (Davies, Pantzopoulos, \& Gray, 2011). Within this context students become both author and audience, enabling a deeper level of interpretation to occur. Ducate et al.'s (2011) study of three wiki projects for foreign language students found that students shifted from being "information 
consumers" to "knowledge producers." This allowed students to have more self-contributing and coconstitutive roles within the wiki community. A survey and analysis of forty seven student perceptions of a wiki used within the area of information technology (IT) also highlighted the benefits of collaborative learning (Feng \& Beaumont, 2010). Students conducted literature reviews using the wiki and the articles were reviewed by peers and tutors. Researchers found that more high level critical comments were left rather than superficial commentary. Importantly, the authors concluded that student ownership of the wiki promoted responsibility, authorial identity and the values of giving credit for intellectual work (Feng \& Beaumont, 2010).

The very public nature of wikis also has an impact on the level of contribution and the standard of the final work that is published. Wikis clearly advise assessors when a page was last accessed, and when an addition was made, making each student highly accountable for their contributions and actions on the site. Furthermore, if fellow-students comprise the audience for a piece of work or assessment, then increased motivation and effort towards a task may be seen (Ducate et al., 2011). Engagement in learning via wikis may also provide 'social acceptance' and a sense of shared culture, which can lead to positive learning outcomes (Yan et al., 2010). The use of wikis also provides an understanding of how students were able to role-model contributions from other co-authors of the site. The ability to engage in knowledge-building can occur frequently as students watch their peers engaging in knowledge constructions, and reconstructions as they work towards the final versions of their projects (Ruth \& Houghton, 2009). Comments by teaching staff or more capable students that are posted on a wiki while a group is contributing to it, and constructing it, can also impact on the detail and level of analysis achieved in each individual's final contribution (Feng \& Beaumont, 2010). Commentary and encouragement from teaching staff can be seen as a valid source of motivation that allows for the role-modelling and creation of more critical student responses (Deng \& Yuen, 2012).

The use of wikis for peer-based assessment and learning reflections have also been highlighted as effective tools for learning within higher education settings (De Wever et al., 2011). De Wever et al.'s (2011) study of 659 students sought to understand their experience of using a wiki for a group-work assignment and subsequent assessment of their fellow students. Students self-assessed using the four categories of contribution, discussion, sources, and social interaction. The researchers concluded that peer assessment using a wiki was reliable and that it is feasible to implement. Self-assessment using a wiki clearly evidences a blur in the definition of both novice and expert as expertise is developed and constructed as part of the knowledge making process (Ruth \& Houghton, 2009).

In light of the initial successes reported as a result of integrating online, interactive media into assessment for tertiary students, we undertook a teaching and learning exercise in the School of Public Health \& Social Work at the Queensland University of Technology (QUT), Brisbane, Australia, to test the potential of a wiki to enhance the learning experiences and academic performance of public health undergraduate students in the Health, Culture, \& Society Unit. Specifically, we set up a wiki via Wikispaces (http://www.wikispaces.com/), and involved over 500 students in individual assessment pieces that were built, created, and displayed publicly on the Health, Culture, and Society Wiki. Thus, both the process and the product of each individual contribution was developed and displayed on the wiki for their peers to reflect upon, and critique. Students wrote research reports on a range of topics including (1) Australia's ability to provide mental health services, (2) women in sport, (3) health risk behaviours amongst Gen Y, (4) the relationship between the Australian political landscape and public health, and (5) the importance of developing and implementing cultural safety within health care services. The students were also required to post, display and analyse a cultural artefact representing their chosen topic. Students chose from music videos, art pieces, poetry, films, documentaries, advertisements, and posters to express core elements of the public health issue being presented and its salience for the health of the Australian population. Finally, students were required to read the work of at least two other students and post questions and comments on the discussion pages associated with the work of each individual contribution.

In this paper, we firstly describe Bandura's social learning theory (SLT) as it applies to the use of wikis for academic assessment in a tertiary education setting. Secondly, we outline the wiki assessment used for the Health, Culture \& Society unit in the School of Public Health \& Social Work at QUT. Thirdly, we detail the methodology of observation and data collection from students regarding their learning processes 
and outcomes. Fourthly, a conceptual model is proposed to illustrate the findings of this process, and finally, benefits and limitations of applying wikis in the higher education context are discussed.

\section{Theoretical framework: Social learning theory in collaborative cyber spaces}

In keeping with the social and collaborative features of Web 2.0 technology, Bandura's SLT was applied to investigate the effectiveness of a wiki as an online tool to improve the academic writing and referencing standards of undergraduate students in this setting. The premise of SLT is that an individual's cognition, behaviour, and social environment continually interact, in a reciprocal, iterative, and cyclic fashion (Bandura, 1977). Bandura argued that human behaviour is not inbuilt as such, but rather is learnt - both via one's own experiences and via the modelling of others in the human social world. By modelling other people's behaviour, an individual begins to cognitively grasp strategies for carrying out a new, or learnt, behaviour. By observing others, Bandura argued, humans develop knowledge which they use to inform their future actions and behaviours. Bandura described this complex, interactive process of social learning as being comprised of four key conceptual elements. These elements were used as the premise underlying the motivation for students to observe, interact, model, and perform their academic work on the wiki to a raised standard as a result of the psychological and behavioural processes outlined below.

\section{Attentional processes}

In order to observe accurately, attention needs to first be given to the behaviour. If the behaviour is seen as attractive, the individual is more likely to give it attention. An individual may be more inclined to pay attention to a modelled behaviour that they will be required to perform publically, rather than privately. All work for this assessment was required for public and shared display with their peers, thus potentially enhancing their motivation to pay close attention to the work and the performance of others.

\section{Retentional processes}

An accurate reproduction of the modelled behaviour may be more likely if the behaviour is immediately imitated, if the observer is repeatedly exposed to the particular behaviour, and/or if the actions required to complete the behaviour are rehearsed, mentally and then overtly. Where immediate imitation is not possible, the observer relies on memory and imagery, as well as verbal instruction. All students were required to post their individual contribution/page onto a shared wiki, thus ensuring constant and repeated exposure to the work of their peers as they developed and produced their own.

\section{Motor reproduction process}

To convert knowledge into behaviour, the required actions need to be cognitively selected and organised, spatially and temporally. The course of action that an individual chooses to take is largely dependent on the anticipated outcome and the confidence that they posses in their own ability to perform the necessary actions (self-efficacy). Self-efficacy will dictate, at least in part, the level of difficulty they wish to tackle, and the amount of effort they wish to invest. Lower-level actions may be rehearsed and carried out first, before moving on to more complex actions required to perform a specific behaviour. During these stages, informative feedback can allow the observer to self-monitor and self-regulate their performance and make adjustments they deem necessary (Bandura, 1977). In addition, such feedback can "improve and sustain" behaviour over the required time (Bandura, 1977). In this exercise, students were permitted to post their pieces over a period of weeks, thus allowing more confident students to post their work first, and 'model' for students who required more time, or an increased number of stages to develop their work. This allowed students to organise their work spatially and temporally according to their perceived selfefficacy. It also allowed for adjustments and improvements to be made over time, and in consultation with their peers. 


\section{Motivational processes}

People are also more likely to model behaviours that lead to pleasing results (Bandura, 1977). Differential reinforcement by models, such as teachers or fellow students, is likely to lead to appropriate behaviour. If a model gives similar feedback to everyone performing the behaviour, regardless of the quality, the behaviour is not imitated well (Bandura, 1977). The provision of models in learning may allow " novices" to accurately carry out a desired behaviour. Indeed, those who lack confidence, or are dependent on others, may benefit the most from models. The provision of a range of models may lead to creativity and innovation. This provision of models was indeed inherent within the wiki assessment and provided motivation and inspiration for those students with less confidence or ability than their peers. The students who modelled early and well were selected as exemplars', receiving public affirmation from the lecturers, and provided to the others as an example of excellence.

Further to this, Bandura has emphasised the Internet as a tool for "self-controlled learning" (Bandura, 2001), as it provides the education sector with an opportunity to reconceptualise the relationship between learning and thinking (Glassman \& Kang, 2011). In contrast to traditional learning processes, which were based on habit, learning with the use of internet technology can be based on discovery and critical thinking (Glassman \& Kang, 2011). A wiki page is one type of internet-based tool that provides opportunity for teaching and learning (Glassman \& Kang, 2011). A wiki is an online collaborative space that multiple users can simultaneously create, edit, delete and attach comments to, instantly - leading to more pragmatic learning (Glassman \& Kang, 2011). Users can collaborate together on a centralised wiki, or may contribute to their own wiki page in a decentralised fashion, that can be accessed by all in their specific wiki community (Greener, 2009). Instant messaging, emails and chatrooms have been used for some time by teachers for their students to this effect. Wiki pages and blogs build on their benefits. However, wikis allow spontaneity and diversity in communication.

Wikis also provide learning diversity by enabling the supplementation of text with graphics, video, and links (Greener, 2009). The use of wikis therefore embrace the notion that learning is as much a social process as it is a personal process. In comparison to traditional teaching tools, a wiki can improve connectivity between people and promote both critical and self-reflective thinking, thus allowing learning to be based on discovery (Glassman \& Kang, 2011). Wikis can also instil a sense of ownership (Glassman \& Kang, 2011), which can be beneficial if a decentralised wiki is used where each user has their own page. However, this can be barrier to learning if a centralised wiki page is used, as students may begrudge their work being altered by another (Glassman \& Kang, 2011), and a similar finding has been found for the use of blogs (Kim, 2008).

Further to this, wikis allow individuals the opportunity to observe exemplars modelled by other students, abstract key elements of a range of exemplars and deduce the actions required to replicate, approximate or creatively extend on these exemplars. Students can start with lower-level tasks, such as creating a page, naming a page, and setting out sub-headings and adding in hyperlinks to related web resources. Feedback can be provided by teachers and students at any stage, and individuals can choose to modify their wiki based on this feedback. However, a downfall of having a vast amount of information readily available is that poor self-regulators can become overwhelmed and fall behind (Bandura, 2001).

\section{The wiki assessment}

The aim of this assessment task was to encourage students to take the skills they had acquired during the course of the unit, including secondary researching and referencing skills, and the application of social theory for the theoretical analysis of a public health issue, and to be able to apply these skills to generate a deeper understanding of the mechanisms creating, connecting, and sustaining people, places, media, and artefacts within their social and cultural environments. The aim of this pedagogical process was to encourage students to observe things within their social worlds, and think critically about them in relation to broader population health and well-being - including being able to take into account an ethical, theoretical, and philosophical analysis of the issues, and to consider the implications of their analysis for equity, inclusion, policy, and implications for different social groups. 
The students were able to select a research project for the Wiki on one of the following topics:

- Green, gold, and blue: How well does Australia deal with mental health in 2011? What is working, what isn't, and what should be done?

- How healthy is Gen Y? What are the key public health issues facing this generation, and what are the driving social and cultural forces behind these issues?

- How culturally 'clued-in' are our primary and emergency service providers when it comes to sex, pain, and death?

- 'Keeping the Bastards Honest': What do Labor, the Liberals, and the Greens bring to the public health table? Who should we vote for from a public health perspective and why?

- Running like a girl: How equitable is the playing field when it comes to women in sport?

\section{The Assessment Task}

Having selected a topic, the students were directed to the established Health, Culture, \& Society Wiki in Wikispaces (http://www.wikispaces.com/) for the unit, which can be viewed in Figure 1. They followed the steps outlined in Figure 1 to generate their own individual research projects on a page at this website.

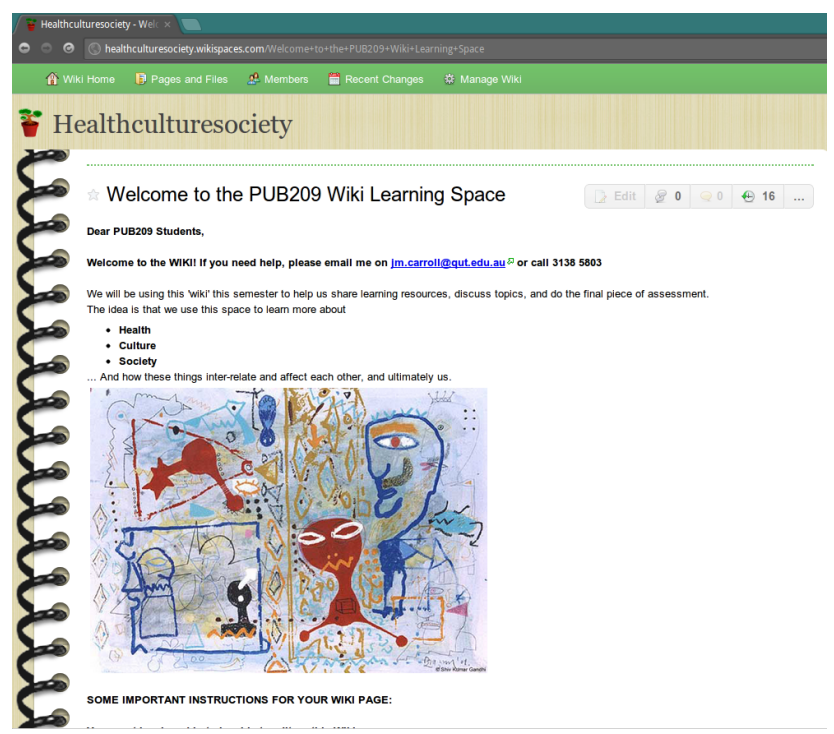

Figure 1. The Health, Culture, \& Society Wiki.

Presentation of a cultural artefact on the wiki

The students' first task involved describing and depicting a cultural artefact. The artefact could be any symbolic item that they had located from within their social/cultural worlds. They were required to describe it, or take a photograph of it, provide a link to its location on Google Maps, provide a link to it on Google, or copy and paste an image of it onto their individual wikipage. Some examples included an art piece, a sculpture, a billboard advertisement, a clothing item, a song from YouTube, a media article, a documentary on TV, and a photograph of something that depicted the public health issue being addressed in the assessment. 
Figures 2, 3, and 4 depict some examples of artefacts used by the students in this assessment piece.

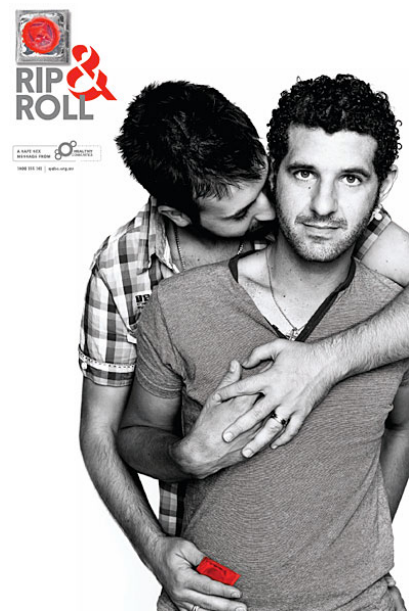

Figure 2. 'Rip and Roll' advertisement for HIV prevention.

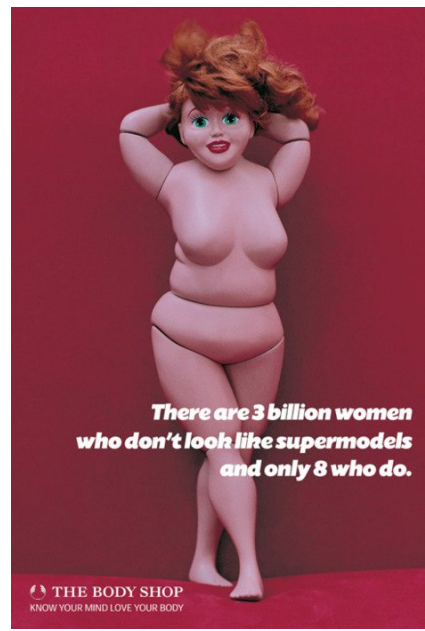

Figure 3. Body Shop advertisement promoting healthy self-image for women.

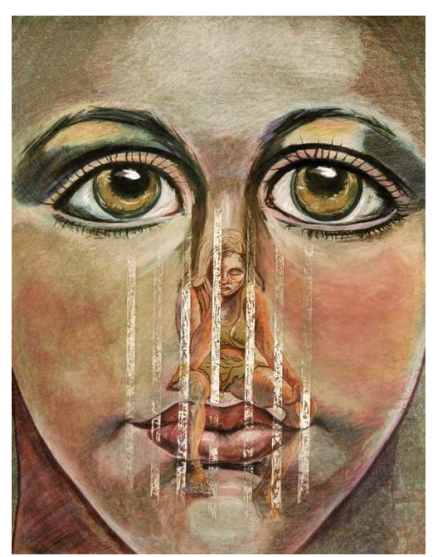

Figure 4. Painting depicting mental illness and depression in particular.

Detailing of the public health issue

The students were to then identify and briefly describe the core public health issue that the cultural artefact represented. This also reflected their chosen topic, and the angles within that topic on which they had selected to focus, for example, the use of medication in the treatment of mental illness. 


\section{Literature review}

The students then conducted a comprehensive and in-depth literature review to demonstrate their knowledge of the latest research and scientific evidence on their chosen topic. Students were required to report on the latest statistics and epidemiological data to highlight the salience of the issue, and to make an argument for a focus on this topic as a priority within the field of public health. They were asked to describe and critique the methodologies employed by researchers to generate their findings, and to locate the limitations (if any) within these studies. They were then required to draw conclusions regarding the key social and cultural determinants of the issue.

Socio-cultural analysis

Students were required to apply social theory, philosophy, and/or a research-based analysis of how and why society and culture were crucial elements in both understanding and redressing the population health issue which they had outlined earlier. They were asked to consider and discuss the group's, movements, social change eras/trends, and cultural forces that both influenced, and were affected by this issue.

\section{Analysis of cultural artefact and reflection exercise}

Finally, the students had to go back to their cultural artefact and describe what it symbolised and represented, how it was a good case in point of their topic/issue, and what it meant to them personally. They were required to reflect on what they had learnt as a result of this assessment piece, and how, if at all, they thought it would affect their future learning and thinking processes.

Together, the students generated a wiki with over 500 contributions of songs, art, photographs, people, conversations, clothing items, sculptures, and research, through which they were able to see how their peers went about researching, evaluating, and analysing their worlds in relation to population health, culture and society.

\section{Research questions}

A number of research questions guided this work

- Can shared cyber spaces be occupied by undergraduate public health students to raise standards in academic research, referencing, and writing skills, and what are the processes via which this occurs?

- Does the interactive process of sharing and comparing assessment items generate collaboration and competition amongst undergraduate students wherein more critically informed arguments are made about public/popular media sources and cultural artefacts depicting issues in contemporary public health?

- What are the implications of this assessment trial for future teaching practices in undergraduate courses?

\section{Data collection}

There were two key methods of data collection undertaken in order to analyse and theorise the learning processes undertaken via this piece of assessment for undergraduate public health students. Firstly, we took screen shots, photographs, and notes about the wiki as it developed. We visually recorded the students' drafts, notes to themselves, and early communication with one another as they developed and refined their own individual pages on the Health, Culture, \& Society Wikispace. Figure 5 shows an example of a student working on the development of their page. We also took screenshots of some of the highest quality pieces and assessments from the wikispace and combined them into a video-clip with a soundtrack for viewing for by the students. The link to the final video of the visual building and finalising of the wiki can be viewed here: Show Me Your Wiki and I'll Show You Mine (YouTube Video, with Soundtrack by Black Gloves) http://www.youtube.com/watch?v=caitIBoCsu0. 


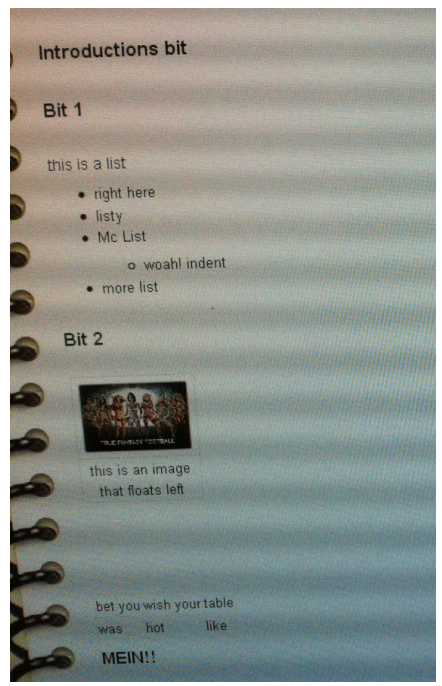

Figure 5. Screenshot of student 'thinking aloud' as they generated their page and learnt how to use the Wiki.

Secondly, and following the completion, submission, marking and return of grades and feedback to the students on their individual wiki page contributions, we asked them the following questions.

- What did you think of the Wiki research project when it was first introduced for assessment?

- What did you like/not like about doing the Wiki research project?

- Do you think your own academic standards were raised as a result of this type of assessment?

- What were some of the factors that affected how you performed in this task?

The questions were kept brief and broad in order to try and get a higher response rate from students who may not have a vested interest in responding to a survey following the completion of assessment and the commencement of their academic break. The questions were open, and worded as broadly as possible to invite open and candid feedback about the learning processes and insights from students. The questions were posted on Blackboard, their shared learning website for the unit, and also individually emailed to the students. We received 22 in-depth written responses from students about their initial reactions to this assessment piece, their strategies for engaging with the task, their learning processes during the task, and their final reflections on the assessment overall.

\section{Data analysis}

We took a social constructionist approach to grounded theory to analyse the data produced by the students in their work and in answer to our research questions (Charmaz, 2000; 2006). This method is adapted from traditional approaches to grounded theory, but with an emphasis on the subjectivity inherent in both the data that is produced, and how that data is produced between the researcher and the participants (Charmaz, 2000; 2006). Due to the reference to Bandura's SLT as a theoretical mechanism for analysing our data and exploring the students' learning processes, a social constructionist methodological approach fitted well with our attention to the interactions that occurred between students in order to inform their learning and academic performance. We conducted a thematic analysis in the first instance, followed by axial coding to explore the relationships between the key concepts arising from the data. We employed SLT to organise the emanating findings from a social constructionist perspective, and to develop theory about how information and communication technologies such as a wiki work to raise standards amongst undergraduate public health students. We paid particular attention to the social dynamics that shaped the students' internal processing and outward performance and academic achievement.

\section{Findings}

The findings are organised by the model below (Figure 6) to highlight the learning processes as described by the students in producing the high quality of academic writing and research we observed in the wiki in the final production. 


\section{Collaboration and competition on a wiki: The praxis of social learning}

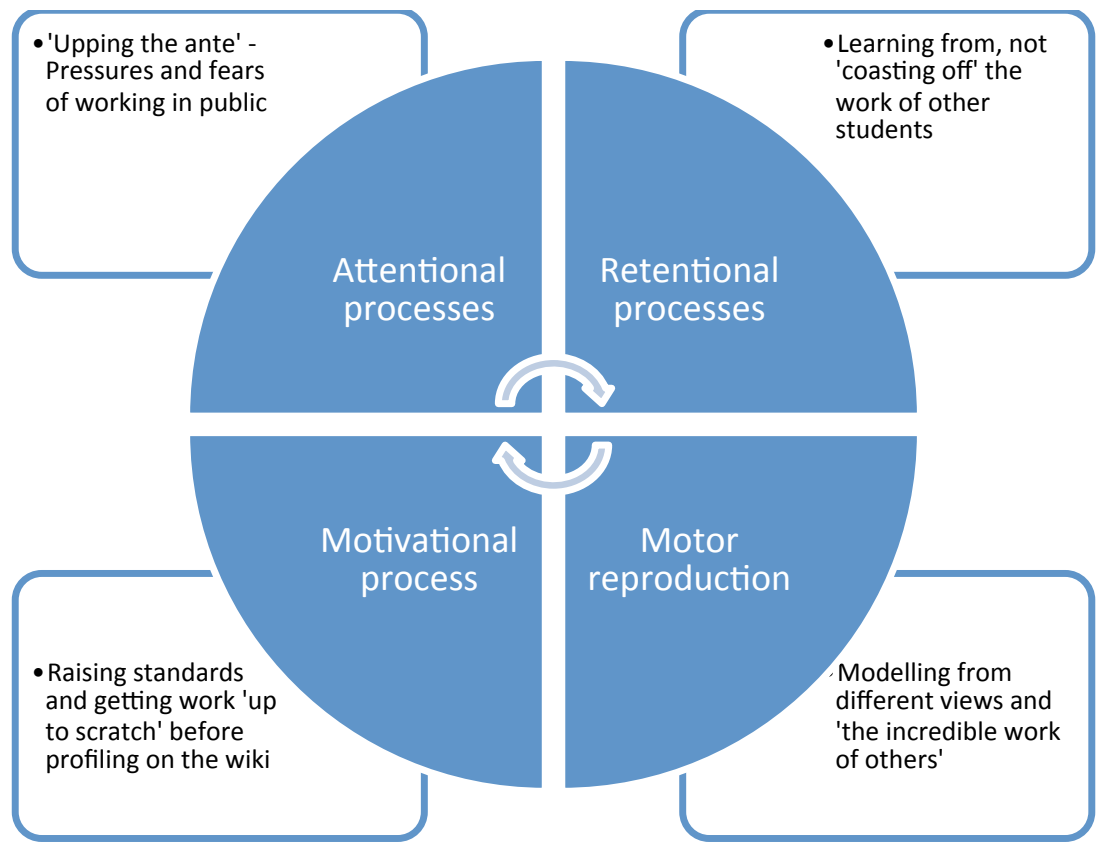

Figure 6. The model developed to organise the findings based on students' descriptions.

\section{Attentional processes}

According to SLT, more attention will be paid to a behaviour that is perceived as appealing or attractive, and which will be required to be performed publically rather than privately. The students stated that while the desire to perform well was indeed present, they were initially intimidated by the demands on them to produce work that would ultimately be visible to the entire student cohort, as these students clearly express:

I didn't like or feel that comfortable with having my name attached to my work for all to see and I was intimidated when I heard the wiki would be able to be accessed by my fellow classmates.

While the task was initially approached with some fear and caution by the students, who were aware of the pressure involved in producing public, rather than private work, this resulted in the initial demands and expectations and motivations being higher, as this student explains:

I guess I felt it created a bit of pressure on myself if other people were going to read it, but really in hindsight that actually worked in my favour, as I put a lot of time and energy into it.

Students generally described this initial period of 'preparing to perform' as being driven by a higher level of ambition to succeed than they normally experience in assessment that will ultimately only be viewed by a teacher:

Having my work on show with the potential for comments from my peers definitely made me up the ante.

\section{Retentional processes}

There was ample opportunity for the students to be exposed to the final products that they were required to produce, as some students began their work early, and there were many examples to watch unfolding and to study during this time. Further to this, the tutors and lecturers posted examples of cultural artefacts and analyses, and examples of how to set up their own individual pages. It was during this time that the cognitive processing around observing, asking, discussing, and reflecting was highest. What was most 
interesting, was that despite students' anticipating that they could simply model, or copy the work of others, this time of observing and processing what was being produced actually made them want to perform even better than their peers, as this student described:

Initially I had thought that doing this assessment might mean that you could coast off those more organized and submitted early, however, it made me want to find something different, and do better than them.

Motor reproduction process

Following the opportunity to observe, ask questions, and plan an approach to their own projects, students began to attempt to post work on the site, and this process was an iterative one of self-reflection and regulation - which they conducted in relation to 'checking-in' with their models and/or peers. During this stage of production, the students were still heavily engaged in observing the work of others around them, as these quotes indicate:

It was great to see others' style and notice the difference in views

[the best part was] Seeing other people's artefacts and wiki's, most of which were incredible

One student reflected on the challenges inherent in making more overt comments, and providing and getting direct feedback on individual efforts. They emphasise that there is an increased challenge in being prepared to openly critique the performance of others, and to be ready to for that critique to be reciprocated:

I found that writing comments on others' work was quite difficult but once I read comments posted on my wiki it was a nice reassurance that I had gotten my message across.

Individual research projects were adjusted towards the generation of a final product as a result of these public interactions with their peers.

\section{Motivational process}

In order to direct students to examples or models of the best work possible, the teaching staff identified the best work being produced and held these up as exemplars for other students to use as a guide. We regularly showed our students pages that had achieved excellence in writing, referencing, and analysis, and told them why we thought these pieces were exceptional. As well as generating a useful guide for performance, this process of rewarding good work instilled a further level of competitiveness amongst students to raise the quality of their work, as this student stated:

My standards were raised because I saw the quality of work posted before I had completed my own wiki. It made me put that extra bit of effort in to get it up to scratch.

Even those students whose self-efficacy was high enough that they were confident to post their work prior to the due date were encouraged to continuously improve their projects based on the exemplars regularly being presented to them, as this student explains:

I put up the page early and edited it regularly based on comments, which was very helpful.

Finally, although it does appear that standards were indeed raised via the processes of collaboration, competition, and modelling, there were other comments by students that indicated the wiki was acting as a mechanism for heightened opportunities to be creative and unique, and deeper learning was occurring as a result of the analysis of a cultural artefact.

For me especially I find things a lot easier when I have a deep interest in it and I thought the way that this was set up meant it was easy to find an area of deep interest and the wiki was a great concept which allowed more freedom in the way that students were able to present their views and ideas. 
While aspects of the projects were modelled on the ability of some students to write well and reference correctly, it also provided the opportunity for students to immerse themselves deeply in their own topics, and via this process of competition, delve more comprehensively into their projects to ensure that learning was sustained. The following quote from a student reflects their keenness to pursue an individual learning goal within a social media forum:

I actually liked the idea of the Wiki as we were able to choose the 'path' of learning we wanted to go down while still seeing what others were doing.

\section{Discussion and conclusions}

Social learning theory (SLT) makes the case that observing others can easily turn into comparing one's own work to that of others' (Bandura, 1977). The resulting sense of competition can be healthy and may lead to feelings of pride, achievement, and personal satisfaction. By providing a standard against which one's own performance can be evaluated, modelled behaviour can induce self-motivation (Bandura, 1977). These processes were highly evident and able to be observed in the work of the Health, Culture, \& Society Wiki project, and were further highlighted in students' accounts of undertaking their individual wiki page projects. The learning that occurred was done socially, publically, collaboratively, and competitively; and via an iterative process wherein students observed and studied each other's' work, and then both imitated and innovated ways of conducting their own projects.

We did observe some initial fear and hesitation to engage in the project, wherein only the students with a heightened sense of self-efficacy were confident to begin posting and sharing their work on this social media forum. It became evident that within this cohort the feedback exposed feelings ranging from intimidation at their work being observed by their contemporaries through to the pragmatism of rising to the challenge of performing at their best because the work would be scrutinized by their peers. However, as predicted in SLT theory, the value the individual students placed on accomplishing the desired behaviour allowed them to overcome such barriers. Bandura suggested that while people will "go to great lengths" to avoid public humiliation (Bandura, 1977), self-regard and self-satisfaction are usually stronger motivators for accomplished performances (Bandura, 1977). By observing how the behaviour of students who were performing well was reinforced by the teaching staff and by their peers, students were able to create new, or alter existing values relating to their own behaviour and performance.

Verification of one's own knowledge is required for effective cognitive functioning (Bandura, 1977). Social verification is one process to do this. By comparing one's own views with that of others, one may change or reinforce their pre-existing judgements. This element of SLT was particularly pertinent for the work conducted by the students in this wiki assessment piece, as students were able to enrich their own perspectives, views, knowledge and expertise on their own topics by having access to the insights of their peers discussing similar issues. They could easily access a range of views and research on a particular topic and compare it to the research they had compiled on their own, thus adding depth and new dimensions to work that is usually done without such social consultation or interaction. A potential trap, however, is that verification can be based on insufficient research or on an inaccurate, yet highly persuasive source of comparison. Mass media, including the multitude of available images readily available on the internet, can be a source of verification of one's own pre-existing views. However, one's pre-existing views will dictate, to some extent, which messages will be absorbed (Bandura, 1977). Students were encouraged to consider all the different views, perspectives, and analyses they encountered in relation to the latest, highest quality research and evidence-based literature.

Overall, the wiki allowed some of the most advantageous elements of social media and information and communication technologies to work in parallel with the pedagogical goals of the teaching staff to ensure deep and sustained learning for students; learning which had been inspired by competition, and informed by lengthy periods of collaboration and iterative reflective processes. The analysis of a cultural artefact encouraged the students to read and understand social and cultural theory as tools for critiquing complex contemporary issues in public health. Bringing an exercise that involved researching the evidence-based literature, applying social theory, and analysing symbolic cultural artefacts within the social media space of a wiki raised the academic writing and research standards of undergraduate students considerably and notably. We attribute much of this success to the attentional, retentional, motor reproduction, and motivational processes outlined by Bandura (1977) in his SLT - and conclude that social media are 
potentially excellent mechanisms to enhance the enjoyment that students gain from, and the concerted commitment they bring to, undergraduate academic assessment.

\section{References}

Bandura, A. (1977). Social Learning Theory. Prentice-Hall, Inc: Englewood Cliffs, N.J.

Bandura, A. (2001). Social cognitive theory: An agentic perspective. Annual Review of Psychology, 52,126.

Charmaz, K. (2000). Grounded theory: Objectivist and constructivist methods. In K. Denzin. \& Y. S. Lincoln (Eds.), Handbook of qualitative research (2nd ed.). Thousand Oaks, CA.: Sage.

Charmaz, K. (2006). Constructing grounded theory: A practical guide through qualitative analysis. Pine Forge Press.

Davies, A., Pantzopoulos, K., \& Gray, K. (2011). Emphasising assessment 'as' learning by assessing wiki writing assignments collaboratively and publicly online. Australasian Journal of Educational Technology, 27(5), 798-812.

Deng, L., \& Yuen, A. (2012). Understanding student perceptions and motivation towards academic blogs: An exploratory study. Australasian Journal of Educational Technology, 28(1), 48-66.

De Wever, B., Van Keer, H., Schellens, T., \& Valcke, M. (2011). Assessing collaboration in a wiki: The reliability of university students' peer assessment. The Internet and Higher Education, 14(4), 201-206.

Ducate, L., Anderson, L., \& Moreno, N. (2011). Wading through the world of wikis: An analysis of three wiki projects. Foreign Language Annals, 44(3), 495-524.

Duffy, P. D., \& Bruns, A. (2006, September). The use of blogs, wikis and RSS in Education: A conversation of possibilities. Paper presented at the Online Learning and Teaching Conference, Brisbane, Australia. Abstract retrieved from http:/eprints.qut.edu.au/5398/

Feng, S., \& Beaumont, C. (2010). Evaluating the use of a wiki for collaborative learning. Innovations in Education and Teaching International, 47(4), 417-431.

Glassman, M., \& Kang, M. J. (2011). The logic of wikis: The possibilities of the web 2.0 classroom. Computer-Supported Collaborative Learning, 6, 93-112.

Greener, S. (2009). Talking online: reflecting on online communication tools. Campus-Wide Information Systems, 26(3), 178-190.

Hemmi, A., S., Bayne, S., \& Land, R.(2009). The appropriation and repurposing of social technologies in higher education. Journal of Computer Assisted Learning, 25, 19-30.

Hodgkinson-Williams, C., Slay, H., \& Siebörger, I. (2008). Developing communities of practice within and outside higher education institutions. British Journal of Educational Technology, 39(3), 433-442.

Hutchinson, A., \& Colwell, J. (2012). Using a wiki to facilitate an online professional learning community for induction and mentoring teachers. Education and Information Technologies, 17(3), 273-289.

Kim, H. N. (2008). The phenomenon of blogs and theoretical model of blog use in educational contexts. Computers and Education, 51, 1342-1352.

Kirkwood. A. (2008). Getting it from the web: Why and how online resources. Journal of Computer Assisted Learning, 24(5), 372-382. 
Knight. J. (2010). Distinguishing the learning approaches adopted by undergraduates in their use of online resources. Active Leaning in Higher Education, 11(1), 67-76.

Ruth, A., \& Houghton, L. (2009). The wiki way of learning. Australasian Journal of Educational Technology, 25(2), 135-152.

Tétard, F., Patokorpi, E., \& Packalén, K. (2009). Using wikis to support constructivist learning: a case study in university education settings. Proceedings of the 42nd Hawaii International Conference on System Sciences (HICSS-42), (pp. 1-10). doi:10.1109/HICSS.2009.499

Wheeler, S., Yeomans, P., \& Wheeler, D. (2008). The good, the bad and the wiki: Evaluating studentgenerated content for collaborative learning. British Journal of Educational Technology, 39(6), 987 995.

Yan, A. Y., Tian, S. W., Vogel, D., \& Kwok, R. C.-W.. (2010). Can learning be virtually boosted? An investigation of online social networking impacts. Computers \& Education, 55(4), 1494-1503.

Corresponding author: Julie-Anne Carroll, jm.carroll@qut.edu.au

Australasian Journal of Educational Technology (C) 2013.

Please cite as: Carroll, J.-A., Diaz, A., Meiklejohn, J., Newcomb, M., \& Adkins, B. (2013). Collaboration and competition on a wiki: The praxis of online social learning to improve academic writing and research in under-graduate students. Australasian Journal of Educational Technology, 29(4), 513-525. 